\title{
ALIMENTAÇÃO DE LEITÕES COM DIETAS CONTENDO SORO DE LEITE FERMENTADO MAIS ZINCO E COBRE ORGÂNICO
}

\author{
PIGLETS FEEDING WITH DIETS CONTAINING MILK FERMENTED AND ZINC \\ AND COPPER ORGANIC
}

\author{
Hauschild, L. ${ }^{1 *}$, Lovatto, P.A. ${ }^{2}$, Lehnen, C.R. ${ }^{2}$, Andretta, I. ${ }^{2}$, Garcia, G.G. ${ }^{2}$ e Daniel, E. ${ }^{1}$ \\ ${ }^{1}$ Faculdade de Ciências Agrárias e Veterinárias. Universidade Estadual Paulista Júlio de Mesquita Filho \\ (UNESP). Jaboticabal, SP. Brasil. *Ihauschild@gmail.com \\ ${ }^{2}$ Departamento de Zootecnia. Universidade Federal de Santa Maria (UFSM). Santa Maria. Rio Grande do \\ Sul. Brasil.
}

\section{PaLAVRAS CHAVE ADICIONAIS}

Lactobacillus acidophilus. Metabólitos. Microminerais. Suínos.

\section{RESUMO}

Um experimento foi realizado com o objetivo de avaliar o desempenho de leitões alimentados com dietas contendo soro de leite fermentado mais $\mathrm{Zn}$ e Cu complexados a aminoácidos. Foram utilizados 64 leitões, meio-irmãos paternos em um delineamento de blocos ao acaso com dois tratamentos. Um tratamento consistiu de uma dieta

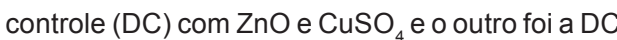
sem $\mathrm{CuSO}_{4}$ e com adição de soro de leite fermentado mais $\mathrm{Zn}$ e $\mathrm{Cu}$ complexados a aminoácidos $\left(\mathrm{SLF}_{\text {Lact }}+\mathrm{ZnCuAA}\right)$. Cada tratamento teve oito repetições e quatro animais por unidade experimental. A presença de $\mathrm{SLF}_{\mathrm{Lact}}+\mathrm{ZnCuAA}$ na dieta de leitões não alterou $(p>0,05)$ o peso vivo dos leitões no experimento. No entanto, o peso vivo diferiu $(p<0,01)$ entre machos e fêmeas $(24,3 v s$. $24,0 \mathrm{~kg}$ ) ao final do experimento. $O$ consumo de ração dos animais do tratamento SLFLact+ZnCuAA foi superior $(p<0,01)$ em $8 \%$ nos primeiros 14 dias $(0,261$ vs. $0,239 \mathrm{~kg} / \mathrm{d}), 15 \%$ de 15 a 28 dias $(0,756$ vs. $0,675 \mathrm{~kg} / \mathrm{d})$ e $12 \%$ de 29 a 42 dias $(1,091$ vs. $0,962 \mathrm{~kg} / \mathrm{d}$ ) em relação ao grupo controle. No período total, o consumo dos animais alimentados com a dieta contendo SLF $_{\text {Lact }}+\mathrm{ZnCuAA}$ foi $11 \%$ superior $(p<0,01)$. $O$ ganho de peso dos animais do tratamento com $\mathrm{SLF}_{\mathrm{Lact}}+\mathrm{ZnCuAA}$ foi superior $(p<0,01)$ em $14 \%$ de 0 a 14 dias $(0,176$ vs. 0,152 $\mathrm{kg} / \mathrm{d}), 11 \%$ de 15 a 28 dias $(0,564 \mathrm{vs} .0,500 \mathrm{~kg} / \mathrm{d})$ e $12 \%$ de 29 a 42 dias $(0,572$ vs. $0,501 \mathrm{~kg} / \mathrm{d})$. O ganho médio diário nos 42 dias de experimento foi

Recibido: 22-9-10. Aceptado:8-6-11.

\section{ADDITIONAL KEYWORDS}

Lactobacillus acidophilus. Metabolites. Microminerals. Pigs.

$12 \%$ superior $(p<0,01)$ nos animais do tratamento com $\mathrm{SLF}_{\text {Lact }}+\mathrm{ZnCuAA}$ comparado ao grupo controle $(0,437$ vs. $0,384 \mathrm{~kg} / \mathrm{d})$. A conversão alimentar do tratamento com $\mathrm{SLF}_{\text {Lact }}+\mathrm{ZnCuAA}$ foi $8 \%$ menor $(p<0,01)$ nos primeiros 14 dias $(1,487$ vs. 1,614$)$. A adição de leite fermentado e Zn e Cu complexados a aminoácidos em dietas contendo óxido de zinco melhora o desempenho de leitões.

\section{SUMMARY}

An experiment was carried out to evaluate the performance of piglets fed rations containing fermented milk and zinc and copper amino acid complex. Sixty four piglets, littermates, were used. The experimental design was based on random block with two dietary treatments. One of these

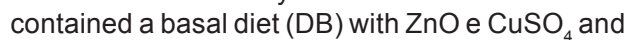
the other one with the $\mathrm{DB}$, but without $\mathrm{CuSO}_{4}$ and with the addition of fermented milk and zinc and copper amino acid complex (SLF Lact $+\mathrm{ZnCuAA})$. There were eight replicate pens per treatment, and pigs were grouped at 4 per pen. The $\mathrm{SLF}_{\text {Lact }}{ }^{+}$ $\mathrm{ZnCuAA}$ didn't not affect $(p>0.05)$ final live weight of the piglets. However, live weight was different $(p<0.01)$ between males and females $(24.3 \mathrm{vs}$. $24.0 \mathrm{~kg}$ ) at end of experiment. The feed intake of animals feed with SLFLact+ZnCuAA was higher $(p<0.01)$ in $8 \%$ in the first 14 days $(0.261$ vs. 0.239 $\mathrm{kg} / \mathrm{d}$ ), $15 \%$ from 15 to 28 days ( $0.756 \mathrm{vs} .0 .675 \mathrm{~kg} /$ 
HAUSCHILD, LOVATTO, LEHNEN, ANDRETTA, GARCIA E DANIEL

d) and $12 \%$ from 29 to 42 days ( $1.091 \mathrm{vs.} 0.962 \mathrm{~kg} /$ d) compared to control group. Daily average feed intake in the experimental period was $11 \%$ higher $(p<0.01)$ for the animals fed with the diet contained $\mathrm{SLF}_{\mathrm{Lat}}+\mathrm{ZnCuAA}$. Weight gain of the treatment with $\operatorname{SLF}_{\text {Lact }}+\mathrm{ZnCuAA}$ was higher $(p<0.01)$ by $14 \%$ from 0 to 14 days $(0.176$ vs. $0.152 \mathrm{~kg} / \mathrm{d}), 11 \%$ from 15 to 28 days ( 0.564 vs. $0.500 \mathrm{~kg} / \mathrm{d}$ ) and $12 \%$ from 29 to 42 days $(0,572$ vs. $0,501 \mathrm{~kg} / \mathrm{d})$. Average daily weight gain of 42 days of trial of treatment with $\mathrm{SLF}_{\text {Lact }}+\mathrm{ZnCuAA}$ was $12 \%$ higher $(p<0.01)$ compared to control group $(0.437 \mathrm{vs} .0 .384 \mathrm{~kg} / \mathrm{d})$. Feed conversion ratio was $8 \%$ lower $(p<0.01)$ for treatment with $\mathrm{SLF}_{\text {Lact }}+\mathrm{ZnCuAA}$ in the first 14 days (1.487 vs. 1.614). Supplementation with fermented milk and zinc and copper amino acid complex in diets containing zinc oxide improved growth performance of weanling piglets.

\section{INTRODUÇÃO}

A redução da idade dos leitões ao desmame vem sendo utilizada com o objetivo de melhorar a produtividade das matrizes (Holtkamp, 1995). Entretanto, devido à imaturidade fisiológica e imunológica dos leitões nessa idade, a prática expõe os animais a fatores que contribuem para incidências de diarréias (Lallès et al., 2004). Dessa forma, novos aditivos nutricionais têm sido estudados com o objetivo de melhorar a adaptação dos leitões a troca de uma alimentação líquida (leite) para sólida (ração).

O uso de níveis elevados de $\mathrm{Zn}$ e $\mathrm{Cu}$ na dieta é uma prática amplamente adotada, com resultados satisfatórios na sanidade e desempenho dos animais (Hahn e Baker, 1993). Essa estratégia, entretanto, devido ao aumento da excreção desses elementos nas fezes e consequentemente no solo, pode comprometer o desenvolvimento de microorganismos e plantas (Jondreville et al., 2003). Neste contexto, ainda existe a necessidade de rever estratégias para alcançar bons resultados sanitários e de desempenho com aspectos ambientais.

Em dietas convencionais, o $\mathrm{Zn}$ ou $\mathrm{Cu}$ alimentar não atendem a exigência desses microminerais para leitões (Jondreville et al., 2003). As fontes inorgânicas têm sido as mais utilizadas nas dietas com inclusões elevadas. Esses níveis têm sido utilizados devido a ação terapêutica no controle da diarréia (Jensen-Waern et al., 1998) e na melhora da morfologia intestinal (Carlson et al., 1999). O Zn aumenta a altura das vilosidades quando utilizado em níveis terapêuticos devido atuar na proliferação celular (Carlson et al., 1998). O uso de fontes orgânicas, como $\mathrm{Zn}$ ou $\mathrm{Cu}$ complexados a aminoácidos pode potencializar a ação do Zn na camada epitelial devido os aminoácidos atuarem como co-transportadores de nutrientes na interface lúmen-enterócitos. Adicionalmente, as fontes orgânicas, como $\mathrm{Zn}$ ou $\mathrm{Cu}$ complexado a aminoácidos, apresentam melhor absorção e biodisponibilidade comparada às inorgânicas (Revy et al., 2002), o que poderia reduzir a excreção desses minerais no ambiente.

Outro aditivo que tem despertado a atenção por seus efeitos imunomoduladores é um composto de metabólitos provenientes da fermentação do soro de leite por uma cepa de Lactobacillus acidophilus (Machnicki et al., 1993). A lactoferrina, um desses metabólitos, quando adicionado nas dietas, melhora a imunidade e morfologia intestinal de leitões (Wang et al., 2006).

O uso desses metabólitos associados a $\mathrm{Zn}$ e $\mathrm{Cu}$ na dieta pode melhorar o desempenho de leitões devido aos efeitos benéficos, mas essa hipótese ainda não foi avaliada em estudos in vivo. Portanto, este trabalho foi conduzido para avaliar o desempenho de leitões alimentados com dietas contendo soro de leite fermentado e $\mathrm{Zn}$ e $\mathrm{Cu}$ complexados a aminoácidos.

\section{MATERIAL E MÉTODOS}

Foram utilizados 64 leitões geneticamente homogêneos, oriundos de cruzamentos industriais, com idade e peso vivo inicial médio de 21 dias e 6,72 quilogramas. Os leitões foram alojados em 16 baias elevadas $(1,50 \times 1,37 \times 0,80 \mathrm{~m}) \mathrm{com}$ 


\section{ALIMENTAÇÃO DE LEITÕES COM SORO DE LEITE FERMENTADO, ZN E CU}

piso parcialmente vazado, equipadas com comedouros semi-automáticos e bebedouros tipo concha. A temperatura da salacreche foi mantida dentro da zona de conforto térmico recomendada para a fase.

O delineamento experimental foi de blocos ao acaso, com dois tratamentos: um composto por uma dieta controle (DC) contendo $\mathrm{ZnO}$ e $\mathrm{CuSO}_{4}$; outro tratamento com a mesma DC, contudo sem $\mathrm{CuSO}_{4}$ e com adição de soro de leite fermentado por Lactobacillus acidophilus $+\mathrm{Zn}$ e $\mathrm{Cu}$ complexados a aminoácidos ( $\mathrm{SLF}_{\text {Lact }}+$ $\mathrm{ZnCuAA}$ ). Cada tratamento teve oito repetições e quatro animais por unidade experimental. Na formação dos blocos foram considerados o peso vivo inicial e o sexo dos animais.

A dieta controle (tabela I) foi formulada para atender as recomendações nutricionais do NRC (1998) para suínos no intervalo de peso entre 6 a $12 \mathrm{~kg}$ (fase 1), 13 a $18 \mathrm{~kg}$ (fase 2) e de 19 a $23 \mathrm{~kg}$ (fase 3 ). $\mathrm{OSLF}_{\text {Lact }}+\mathrm{ZnCuAA}$ foi adicionado somente nas fases 1 ( 0 a 14 dias) e 2 (14 a 28 dias). Nas dietas com $\mathrm{SLF}_{\text {Lact }}+\mathrm{ZnCuAA}$ não foi adicionado $\mathrm{CuSO}_{4}$ e foi reduzido em $1,5 \%$ o nível de lactose. $\mathrm{Na}$ fase 3 (29 a 42 dias), quando as dietas não continham ( $\left.\mathrm{SLF}_{\mathrm{L}}+\mathrm{ZnCuAA}\right)$, foi adicionado somente 400 e 800 ppm de ZnAA e $\mathrm{CuAA}$. O $\mathrm{SLF}_{\text {Lact }}+\mathrm{ZnCuAA}$ é um aditivo nutricional microbiológico produzido através da fermentação do soro de leite por uma cepa específica de Lactobacillus acidophilus. A lactoferrina, uma glicoproteína multifuncional, é um dos principais metabólitos presente neste aditivo. Nesse composto também são adicionados complexos de $\mathrm{Zn}$ e $\mathrm{Cu}$ com aminoácidos resultante de um sal solúvel de metal com um aminoácido (AAFCO, 2003). O perfil nutricional do aditivo $\mathrm{SLF}_{\mathrm{Lact}}+\mathrm{ZnCuAA}$ está apresentado na tabela II. Os animais receberam alimentação à vontade e tiveram livre acesso à água.

Os dados de ganho de peso foram obtidos por pesagens semanais individuais dos animais. O consumo médio diário de ração
Tabela I. Ingredientes e composição calculada da dieta controle. (Ingredients and calculated composition of the control diet).

\begin{tabular}{|c|c|c|c|}
\hline \multirow{2}{*}{$\begin{array}{l}\text { Ingredientes } \\
\text { Milho }\end{array}$} & \multicolumn{3}{|c|}{ Fase 1 Fase 2 Fase 3} \\
\hline & 32,43 & 44,43 & 61,12 \\
\hline Milho pré-cozido & 19,07 & 7,90 & 1,93 \\
\hline Farelo de soja & 12,00 & 25,00 & 31,80 \\
\hline Óleo vegetal & 1,00 & 1,00 & 2,00 \\
\hline Soja micronizada & 14,00 & 7,40 & - \\
\hline Açúcar & 2,00 & 2,00 & - \\
\hline PL $70 \%$ lactose $^{1}$ & 6,07 & 5,00 & - \\
\hline PL $40 \%$ lactose $^{2}$ & 10,00 & 4,00 & - \\
\hline Premix $^{3}$ & 1,34 & 2,00 & 2,35 \\
\hline Antibióticos ${ }^{4}$ & 0,08 & 0,08 & 0,02 \\
\hline Acidificante $^{5}$ & 0,40 & 0,40 & 0,40 \\
\hline L-lisina & 0,57 & 0,25 & 0,17 \\
\hline DL-metionina & 0,28 & 0,13 & 0,08 \\
\hline L-treonina & 0,24 & 0,09 & 0,06 \\
\hline L-triptofano & 0,05 & - & - \\
\hline Óxido de zinco 75\% & 0,40 & 0,25 & - \\
\hline Sulfato de cobre $25 \%$ & 0,07 & 0,07 & 0,07 \\
\hline \multicolumn{4}{|l|}{ Composição calculada } \\
\hline $\mathrm{EM}, \mathrm{kcal} / \mathrm{kg}$ & 3265 & 3265 & 3265 \\
\hline Proteína bruta, \% & 22,500 & 21,02 & 20,30 \\
\hline Cálcio, \% & 0,906 & 0,886 & 0,946 \\
\hline Fósforo total, \% & 0,682 & 0,653 & 0,650 \\
\hline Fósforo disponivel, \% & 0,481 & 0,444 & 0,425 \\
\hline Lisina, \% & 1,260 & 1,118 & 1,172 \\
\hline Metionina + cistina, \% & 0,720 & 0,580 & 0,625 \\
\hline Metionina, \% & 0,499 & 0,340 & 0,327 \\
\hline Treonina, \% & 0,810 & 0,721 & 0,803 \\
\hline
\end{tabular}

EM: Energia metabolizável. ${ }^{1}$ Produto comercial: Nuklospray K-21; ${ }^{2}$ Produto comercial: Nuklospray K-43; ${ }^{3}$ remix mineral, vitamínico e protéico. Quantidades por kg de alimento nas fases 1, 2 (F2) e 3 (F3): I: 3, 8 (F2) e 8 mg (F3); Co: 2 mg ; Fe: 200 , 500 (F2) 1320 mg (F3); Mn: 80, 200 (F2) e 320 mg (F3); Vit. A: 30 000, 60000 (F2) e 160000 UI (F3); Vit. D3: 6000, 12000 (F2) e 30000 UI (F3); Vit. E: 150, 300 (F2) e 1000 mg (F3); Vit. K3: 6, 12 (F2) e 40 mg (F3); Vit. B12: 100, 200 (F2) e 400 mg (F3); Ácido nicotínico: 90, $180 \mathrm{mg}$ (F2); Ácido fólico: 9, 18 (F2) e 16 mg (F3); Biotina: 0,6, 1,2 (F2) e 2 mg (F3); Ácido pantotênico: 30, 60 (F2) e 200 mg; Proteína bruta: $17,14 \%(\mathrm{~F} 2) .{ }^{4}$ Sulfato de colistina nas fases 1 e 2: $625 \mathrm{mg} / \mathrm{kg}$; Halquinol em todas as fases: $200 \mathrm{mg} / \mathrm{kg}$; ${ }^{5}$ Produto comercial: Calprona PP6. 
HAUSCHILD, LOVATTO, LEHNEN, ANDRETTA, GARCIA E DANIEL

Tabela II. Perfil nutricional do soro de leite fermentado com Lactobacillus acidophilus com adição de zinco e cobre complexados a aminoácidos $\left(S L F_{\text {Lact }}+\mathrm{ZnCuAA}\right)$. (Nutritional profile of whey fermented with Lactobacillus acidophilus with the addition of zinc and copper complexed to amino acids $-\mathrm{SLF}_{\text {Lact }}+\mathrm{ZnCuAA}$ ).

\begin{tabular}{lc}
\hline Nutrientes & SLF $_{\text {Lact }}+$ ZnCuAA \\
\hline Matéria seca, \% & 92,0 \\
Proteína, \% & 16,0 \\
Extrato etéreo, \% & 1,5 \\
Lactose, \% & 15,0 \\
pH & 3,7 a 4,3 \\
Ácido lático & 5,0 \\
Energia metabolizável, kcal/kg & 2755 \\
Zinco, ppm & 2000 \\
Cobre, ppm & 4000 \\
\hline
\end{tabular}

foi obtido pela pesagem da ração fornecida menos os resíduos presentes nos comedouros. Os dados de peso vivo foram analisados por medidas repetidas no tempo através do procedimento Proc Mixed do SAS. No modelo foram inseridos o efeito de tratamento, bloco, sexo, período e tratamento*período. $\mathrm{Na}$ análise de medidas repetidas no tempo foi utilizada a estrutura de covariância autoregressiva (AR). Os dados de consumo médio de ração, ganho de peso e conversão alimentar obtidos foram submetidos à análise de variância incluindo no modelo os efeitos dos tratamentos, bloco e sexo. As análises estatísticas foram reali- zadas através do SAS versão 8.0 (SAS, 2000).

\section{RESULTADOSEDISCUSSÃO}

Os resultados de peso vivo dos leitões alimentados com a dieta controle e dieta contendo soro de leite fermentado e zinco e cobre complexados a aminoácidos $\left(\mathrm{SLF}_{\text {Lact }}{ }^{+}\right.$ $\mathrm{ZnCuAA)} \mathrm{estão} \mathrm{apresentados} \mathrm{na} \mathrm{tabela} \mathrm{III.}$ A presença de $\mathrm{SLF}_{\mathrm{Lact}}+\mathrm{ZnCuAA}$ na dieta de leitões não alterou $(\mathrm{p}>0,05)$ o peso vivo dos leitões no experimento. No entanto, o peso vivo diferiu $(\mathrm{p}<0,01)$ entre machos e fêmeas $(24,3$ vs. $24,0 \mathrm{~kg})$ ao final do experimento.

No metabolismo proteico o $\mathrm{Zn}$ atua de forma positiva na síntese de DNA, devido ao seu efeito primário na expressão genética (Underwood e Suttle, 1999). No entanto, níveis elevados de $\mathrm{Zn}$ na dieta ou no plasma não implicam necessariamente em um aumento na síntese proteica. Todo o $\mathrm{Zn}$ excedente, não utilizado pelo organismo, é excretado nas fezes e na urina através de mecanismos homeostáticos (Poulsen e Larsen, 1995). Nesse estudo, em ambos os tratamentos, os níveis suplementados atendem a exigência de $\mathrm{Zn}$ para a síntese de proteína o que pode explicar não ter havido diferenças entre os tratamentos quanto ao peso vivo. Outros estudos também não observaram diferenças no peso vivo de leitões alimentados com dietas contendo $\mathrm{Zn}$ ou $\mathrm{Cu}$ complexados a aminoácidos (Coffey et al., 1994; Muniz et al., 2010) O

Tabela III. Peso vivo de leitões alimentados com a dieta controle e da dieta contendo soro de leite fermentado mais zinco e cobre complexados a aminoácidos $\left(S L F_{L a c t}+Z n C u A A\right)$. (Body weight of piglets fed with control diet and diet containing fermented whey plus zinc and copper complexed to amino acids $-\mathrm{SLF}_{\text {Lact }}+\mathrm{ZnCuAA}$ ).

\begin{tabular}{ccclc}
\hline Variáveis & Controle & SLF $_{\text {Lact }}+Z n C u A A$ & \multicolumn{2}{c}{ Nível de probabilidade } \\
\hline $\begin{array}{c}\text { Peso vivo, kg } \\
\text { Inicial }\end{array}$ & & & Tratamento & $\mathrm{p}=0,096$ \\
$14 \mathrm{~d}$ & 6,68 & 6,77 & Bloco & $\mathrm{p}=0,815$ \\
$28 \mathrm{~d}$ & 8,81 & 9,23 & Sexo & $\mathrm{p}<0,001$ \\
$42 \mathrm{~d}$ & 15,77 & 17,13 & & \\
& 23,15 & 25,14 & Erro padrão das médias & 0,208 \\
\hline
\end{tabular}

Archivos de zootecnia vol. 61, núm. 233, p. 74. 
estudo de Carlson et al. (2004) não mostrou diferenças no peso de leitões recém desmamados alimentados com dietas contendo níveis crescentes de Zn (50 a 800 ppm) complexados a uma proteína ou contendo $2000 \mathrm{ppm}$ de $\mathrm{Zn}$ na forma de $\mathrm{ZnO}$ (inorgânica) ao final de 28 dias experimentais.

O desempenho zootécnico dos leitões alimentados com a dieta controle e a dieta contendo $\mathrm{SLF}_{4}+\mathrm{ZnCuAA}$ está apresentado na tabela IV. Houve diferença entre os tratamentos $(\mathrm{p}<0,01)$ no consumo de ração em todas as fases e no período total. Nos primeiros 14 dias, o consumo da dieta contendo $\mathrm{SLF}_{\mathrm{Lact}}+\mathrm{ZnCuAA}$ pelos leitões foi $8 \%$ superior $(\mathrm{p}<0,01)$ comparada à dieta controle (0,261 vs. $0,239 \mathrm{~kg} / \mathrm{d})$. Entre os $15 \mathrm{e}$ 28 dias de experimento, o consumo de ração dos animais alimentados com a dieta contendo $\mathrm{SLF}_{\text {Lact }}+\mathrm{ZnCuAA}$ foi $11 \%$ superior $(\mathrm{p}<0,01)$ em relação ao tratamento controle $(0,756 v s .0,675 \mathrm{~kg} / \mathrm{d})$. Dos 29 aos 42 dias, o consumo de ração dos animais alimentados com a dieta contendo SLF $_{\text {Lt }}+$
$\mathrm{ZnCuAA}$ foi $12 \%$ superior $(\mathrm{p}<0,01)$ comparado ao grupo controle $(1,091 \mathrm{vs} .0,962 \mathrm{~kg} /$ d). No período total o consumo dos animais alimentados com a dieta contendo $\mathrm{SLF}_{\text {Lact }}+$ $\mathrm{ZnCuAA}$ foi $11 \%$ superior $(\mathrm{p}<0,01)$.

$\mathrm{O}$ ganho de peso dos animais do tratamento contendo $\mathrm{SLF}_{\text {Lact }}+\mathrm{ZnCuAA}$ foi $14 \%$ superior $(p<0,01)$ de 0 a 14 dias $(0,176$ vs. $0,152 \mathrm{~kg} / \mathrm{d})$, em $11 \%$ de 15 a 28 dias $(0,564$ vs. $0,500 \mathrm{~kg} / \mathrm{d})$ e em $12 \%$ de 29 a 42 dias $(0,572$ vs. $0,501 \mathrm{~kg} / \mathrm{d})$. O ganho de peso médio dos animais alimentados com dietas contendo $\mathrm{SLF}_{\text {Lact }}+\mathrm{ZnCuAA}$ durante os 42 dias foi $12 \%$ superior $(p<0,01)$ comparado ao grupo controle $(0,437 v s .0,384 \mathrm{~kg} / \mathrm{d})$. A conversão alimentar foi $8 \%$ melhor $(p<0,01)$ para os leitões alimentados com dietas contendo $\mathrm{SLF}_{\text {Lact }}+\mathrm{ZnCuAA}$ comparada à dieta controle nos primeiros 14 dias de experimento $(1,487$ vs. 1,614). Nas demais fases e no período total não houve diferença $(p>0,05)$ entre os tratamentos.

O Zn, pelo papel importante na proliferação celular (Revy et al., 2003), aumenta

$\overline{\text { Tabela IV. Desempenho zootécnico de leitões alimentados com uma dieta controle e outra }}$ contendo soro de leite fermentado e zinco e cobre complexados a aminoácidos $\left(S L F_{\text {Lact }}+Z n C u A A\right)$. (Performance of piglets fed with control diet and diet containing fermented whey plus zinc and copper complexed to amino acids $-\mathrm{SLF}_{\text {Lact }}+\mathrm{ZnCuAA}$ ).

\begin{tabular}{|c|c|c|c|c|}
\hline Variáveis & Controle & $\mathrm{SLF}_{\text {Lact }}+\mathrm{ZnCuAA}$ & $\mathrm{Epr}^{1}$ & Efeito \\
\hline \multicolumn{5}{|c|}{ Consumo de ração, kg/dia } \\
\hline $0-14 \mathrm{~d}$ & 0,239 & 0,261 & 0,011 & $\mathrm{~T}^{* *} ; \mathrm{B} ; \mathrm{S}$ \\
\hline $15-28 d$ & 0,675 & 0,756 & 0,027 & $\mathrm{~T}^{* *} ; \mathrm{B} ; \mathrm{S}^{* *}$ \\
\hline $29-42 d$ & 0,962 & 1,091 & 0,032 & $\mathrm{~T}^{* *} ; \mathrm{B} ; \mathrm{S}^{\star *}$ \\
\hline $1-42 d$ & 0,625 & 0,703 & 0,022 & $\mathrm{~T}^{* *} ; \mathrm{B} ; \mathrm{S}^{*}$ \\
\hline \multicolumn{5}{|c|}{ Ganho de peso, kg/dia } \\
\hline $0-14 \mathrm{~d}$ & 0,152 & 0,176 & 0,009 & $T^{\star \star} ; B ; S$ \\
\hline $15-28 d$ & 0,500 & 0,564 & 0,022 & $\mathrm{~T}^{* *} ; \mathrm{B} ; \mathrm{S}^{*}$ \\
\hline $29-42 d$ & 0,501 & 0,572 & 0,019 & $\mathrm{~T}^{* *} ; \mathrm{B} ; \mathrm{S}^{* *}$ \\
\hline $1-42 d$ & 0,384 & 0,437 & 0,013 & $\mathrm{~T}^{* *} ; \mathrm{B} ; \mathrm{S}^{* *}$ \\
\hline \multicolumn{5}{|c|}{ Conversão alimentar } \\
\hline $0-14 \mathrm{~d}$ & 1,614 & 1,487 & 0,076 & $\mathrm{~T}^{* *} ; \mathrm{B} ; \mathrm{S}$ \\
\hline $15-28 d$ & 1,364 & 1,34 & 0,032 & T; B; S \\
\hline $29-42 d$ & 1,964 & 1,925 & 0,085 & $\mathrm{~T} ; \mathrm{B} ; \mathrm{S}$ \\
\hline $1-42 d$ & 1,652 & 1,585 & 0,055 & $\mathrm{~T} ; \mathrm{B} ; \mathrm{S}$ \\
\hline
\end{tabular}

${ }^{1}$ Erro padrão das médias, $\mathrm{T}=$ tratamento; $\mathrm{B}=$ bloco; $\mathrm{S}=\mathrm{sexo} ;{ }^{*} \mathrm{p}<0,05 ;{ }^{* *} \mathrm{p}<0,01$. 
HAUSCHILD, LOVATTO, LEHNEN, ANDRETTA, GARCIA E DANIEL

a altura das vilosidades quando utilizado em níveis terapêuticos (Carlson et al., 1998). A lactoferrina, além de aumentar a altura das vilosidades, também reduz a profundidade das criptas quando adicionada em dietas de leitões (Wang et al., 2006). Adicionalmente, esse metabólito possui outras funções fisiológicas, como proteção contra infecções intestinais (Dial et al., 1998) e regulação da função imune (Lee, 1998). Esse efeito positivo na morfologia ou imunidade de cada componente da dieta contendo $\mathrm{SLF}_{\mathrm{Lact}+}$ $\mathrm{ZnCuAA}$ contribui de forma aditiva no aumento do consumo e melhora do desempenho dos animais. Resultados semelhantes ao do presente estudo foram encontrados por Wang et al. (2007), que constataram aumentos na ordem de $34 \%$ no ganho de peso diário, $17 \%$ no consumo diário de ração e melhora de $12,8 \%$ na conversão alimentar de leitões recém desmamados alimentados com uma dieta contendo $1 \mathrm{~g} / \mathrm{kg}$ de lactoferrina. Os mesmos autores atribuíram esses resultados à microbiologia e morfologia do intestino delgado, que também foram positivamente influenciadas por esse metabólito. Em estudos semelhantes, além de conferir melhor desempenho, a lactoferrina melhorou a imunidade de leitões recém desmamados, indicando ser uma alternativa ao uso de antibióticos (Wang et al., $2006 \mathrm{e}$ Shan et al., 2007).

A proibição pela União Europeia do uso de antibióticos como promotores de crescimento em dietas para leitões a partir

\section{BIBLIOGRAFIA}

AAFCO. 2003. Association of American Feed Control Official. Official publication 2003. <http:/ /www.aafco.org/Home/OrderAAFCOPublica tions> (02/12/2009).

Carlson, M.S., Boren, C.A., Wu, C., Huntington, C.E., Bollinger, D.W. and Veum, T.L. 2004. Evaluation of various inclusion rates of organic zinc either as polysaccharide or proteinate complex on the growth performance, plasma and excretion of nursery pigs. J Anim Sci, 82: 1359-1366. de 1997 intensificou as pesquisas na busca de novas alternativas (Casewell et al., 2003). $\mathrm{O}$ uso de dietas integrando diferentes aditivos nutricionais pode ser uma prática que permita substituir parcial ou totalmente os antibióticos. $\mathrm{O}$ uso de $\mathrm{Zn}$ e $\mathrm{Cu}$ suplementados nas dietas já é uma prática amplamente utilizada. Os níveis e as fontes adequadas, contudo, ainda são motivos de discussões quando se refere a aspectos ambientais. Nesse estudo, foi possível reduzir o nível de $\mathrm{Cu}$ na dieta com $\mathrm{SLF}_{\text {Lact }}+\mathrm{ZnCuAA}$ e manter bons resultados de desempenho.

\section{CONCLUSÕES}

O uso de soro de leite fermentado e zinco e cobre complexados a aminoácidos em dietas contendo óxido de zinco aumenta o consumo de ração e ganho de peso de leitões na creche e melhora a conversão alimentar nos primeiros 14 dias de creche.

\section{AGRADECIMENTOS}

Os autores agradecem à Zinpro Corporation (Eden Praire, $\mathrm{MN}$ ) pelo financiamento parcial do projeto. À Comissão de Aperfeiçoamento de Pessoal de Nível Superior (CAPES) pelas bolsas de mestrado à Ines Andretta e de doutorado à Cheila Roberta Lehnen. Ao Conselho Nacional para o Desenvolvimento Científico e Tecnológico (CNPq) pela concessão de bolsa de Produtividade em Pesquisa a Paulo Alberto Lovatto.

Carlson, M.S., Hill, G.M. and Link, J.E. 1999. Earlyand traditionally weaned nursery pigs benefit from phase-feeding pharmacological concentrations of zinc oxide: effect on metallothionein and mineral concentrations. J Anim Sci, 77: 1199-1207.

Carlson, M.S., Hoover, S.L., Hill, G.M., Link, J.E. and Turk, J.R. 1998. Effect of pharmacological zinc on intestinal metallothionein concentration and morphology in the nursery pig. J Anim Sci, 76: 57 


\section{ALIMENTAÇÃO DE LEITÕES COM SORO DE LEITE FERMENTADO, ZN E CU}

Casewell, M., Friis, C., Marco, E., McMullin, P. and Phillips, I. 2003. The European ban on growthpromoting antibiotics and emerging consequences for human and animal health. $J$ Antimicrob Chemoth, 52: 159-161.

Coffey, R.D., Cromwell, G.L. and Monegue, H.L. 1994. Efficacy of a copper-lysine complex as a growth promotant for weanling pigs. J Anim Sci, 72: 2880-2886.

Dial, E.J., Hall, L.R., Serna, H., Romero, J.J., Fox, J.G. and Lichtenberger, L.M. 1998. Antibiotic properties of bovine lactoferrin on Helicobacter pylori. Digest Dis Sci, 43: 2750-2756.

Hahn, J.D. and Baker, D.H. 1993. Growth and plasma zinc responses of young pigs fed pharmacologic levels of zinc. J Anim Sci, 71: 3020-3024.

Holtkamp, D.J. 1995. Productivity gains related to segregated early weaning in pigs. In: Annual Meeting of American Association of Swine Practitioners. Nebraska. pp. 217-223.

Jensen-Waern, M., Melin, L., Lindberg, R., Johannisson, A., Petersson, L. and Wallgren, $P$ 1998. Dietary zinc oxide in weaned pigs effects on performance, tissue concentrations, morphology, neutrophil functions and faecal microflora. Res Vet Sci, 64: 225-231.

Jondreville, C., Revy, P.S. and Dourmad, J.Y. 2003. Dietary means to better control the environmental impact of copper and zinc by pigs from weaning to slaughter. Livest Prod Sci, 84 147-156.

Lallès, J.P., Boudry, G., Favier, C., Le Floc'h, N., Luron, I., Montagne, L., Oswald, I.P., Pié, S., Piel, C. and Sève, B. 2004. Gut function and dysfunction in young pigs: physiology. Anim Res, 53: 301-316.

Lee, W.L. 1998. The protective effects of lactoferrin feeding against endotoxin lethal shock in germfree piglets. Infect Immun, 66: 1421-1426.

Machnicki, M., Zimecki, M. and Zagulski, T. 1993. Lactoferrin regulates the release of tumour necrosis factor alpha and interleukin 6 in vivo. Int J Exp Pathol, 74: 433-439.

Muniz, M.H.B., Berto, D.A., Hauptli, L., Fracarolli, C., Neto, M.A.T., Tamassia, L.F.M. e Wechsler, F.S. 2010. Fontes orgânicas e inorgânicas de zinco e cobre como melhoradores de desempenho em leitões desmamados. Rev Bras Zootecn, 39:1999-2005.

NRC. 1998. National Research Council. Nutrient requirements of swine. National Academy of Science. Washington. 189 pp.

Poulsen, H.D. and Larsen, T. 1995. Zinc excretion and retention in growing pigs fed increasing levels of zinc oxide. Livest Prod Sci, 43: 235242.

Revy, P.S., Jondreville, C., Dourmad, J.Y. et Nys, Y. 2003. Le zinc dans l'alimentation du porc: oligoélément essentiel et risque potentiel pour I'environnement. INRA Prod Anim, 16: 3-18.

Revy, P.S., Jondreville, C., Dourmad, J.Y., Guinotte, F. and Nys, Y. 2002. Bioavailability of two sources of zinc in weanling pigs. Anim Res, 51: 315-326.

SAS. 2000. Statistical analysis system user's guide. Release 8.0. SAS Inst. Cary, N.C. USA.

Shan, T., Wang, Y., Liu, J. and Xu, Z. 2007. Effect of dietary lactoferrin on the immune functions and serum iron level of weanling piglets. J Anim Sci, 85: 2140-2146.

Underwood, E.J. and Suttle, N.F. 1999. The mineral nutrition of livestock. CABI Publishing. Wallingford. UK. $614 \mathrm{pp}$.

Wang, Y., Shan, T., Xu, Z., Liu, J., Feng, J. and Wang, Z.Q. 2007. Effects of the lactoferrin (LF) on the growth performance, intestinal microflora and morphology of weanling pigs. Anim Feed Sci Tech, 135: 263-272.

Wang, Y., Shan, T., Xu, Z., Liu, J. and Feng, J. 2006 Effect of lactoferrin on the growth performance, intestinal morphology, and expression of PR-39 and protegrin-1 genes in weaned piglets. J Anim Sci, 84: 2636-2641. 